\title{
INTEGRATION OF INS AND GNSS FOR GRAVIMETRIC APPLICATION WITH UAS
}

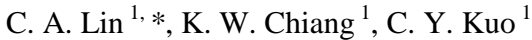 \\ ${ }^{1}$ Dept. of Geomatics, National Cheng Kung University, Tainan, Taiwan (R.O.C.) - (p68011068, kwchiang, \\ kuo70)@mail.ncku.edu.tw
}

Commission I, WG I/6

KEY WORDS: INS, GNSS, UAS, ZUPT, Gravity disturbance

\begin{abstract}
:
The integration based on Inertial Navigation System (INS) and Global Navigation Satellite System (GNSS) has been successfully developed in observing the gravity. Therefore, this study integrates a navigation-grade INS, iNAV-RQH from iMAR and GNSS from NovAtel to develope an Unmanned Aerial System (UAS) using unmanned helicopter for gravimetric application. The advantages include its good flexibility, and it is an intermediate system between the airborne and terrestrial survey in terms of the coverage and spatial resolution. On the other hand, based on the characteristics of vertical take-off and landing, in addition to the kinematic mode as the measure method in the acquisition of gravimetric results, the Zero Velocity Update (ZUPT) mode is implemented as a novel method with the developed UAS. The preliminary results in kinematic mode show that the internal accuracies of horizontal and vertical gravity disturbance at crossover points are approximately 6-11 mGal and $4 \mathrm{mGal}$, respectively, with a $0.5-\mathrm{km}$ resolution. As expected, the accuracy in down component is higher than that in horizontal components because the orientation errors could cause large error in horizontal components. Moreover, the repeatability in ZUPT mode is evaluated with accuracies of approximately 2-3 mGal. The capability of developed UAS for gravimetric application has been demonstrated through various scenarios in this study.
\end{abstract}

\section{INTRODUCTION}

Inertial Navigation System (INS) and Global Navigation Satellite System (GNSS) have been integrated for various applications over the past few decades. INS contains three-axis accelerometers and three-axis gyroscopes used to determine the motion of the vehicle. Benefiting from the improvements in IMU hardware and GNSS kinematic positioning technology, combining data from the INS/GNSS gravimetry for moving platforms has shown great performance in estimating gravity (Kleusberg et al., 1990; Glennie, 1999; Kwon and Jekeli, 2001; Kreye and Hein, 2003). The INS/GNSS gravimetry is based on a vehicle to measure the gravity changes and estimate gravity disturbance $(\delta \mathrm{g})$, which is the difference between actual gravity and normal gravity. The scalar approach in navigation frame (n-frame) according to the IMU and GNSS data (Schwarz, 2006):

$\delta g^{n}=a_{u}{ }^{n}-\ddot{X}_{u}{ }^{n}-\gamma^{n}+\left(\frac{V_{e}}{R_{N}+h}+2 \omega_{E} \cos \varphi\right) V_{e}+\frac{V_{n}{ }^{2}}{R_{M}+h}$

where $a_{u}$ is the up component of the IMU-measured dynamic acceleration, $\ddot{X}_{\mathrm{u}}$ is GNSS-derived kinematic acceleration, $\gamma$ is the normal gravity, $\mathrm{V}_{\mathrm{e}}$ and $\mathrm{V}_{\mathrm{n}}$ are the east and north components of the vehicle's velocity, respectively, $\omega_{\mathrm{E}}$ is the rotation rate of Earth, $h$ and $\varphi$ are the ellipsoidal height and geodetic latitude, and $\mathrm{R}_{\mathrm{N}}$ and $\mathrm{R}_{\mathrm{M}}$ are prime and meridian curvature radii, respectively. The sum of the fourth and fifth terms at the right side is usually called the Eötvös correction, which results from the rotation of the reference coordinate frame. The scalar approach only provides the gravity disturbance in the vertical component according to Equation (1). Based on this drawback, the algorithm for vector gravimetry determination is presented in this study to estimate the gravity disturbance vector in all three components. Furthermore, all calculations are carried out in inertial frame (i-frame), which can reduce the complex computations for the Eötvös correction (Kwon and Jekeli, 2001).

The INS/GNSS airborne gravimetry is an effective method to survey local gravity in a relatively short time. However, there are some shortcomings of present airborne gravimetry. The cost for renting an aircraft to conduct aerial experiment is high and there are strict regulations and complicated procedures for obtaining a permit to conduct a flight mission in most countries. In addition, because of the attenuation of the gravity field with altitudes, detecting short wavelength gravity signals has become a challenge. In order to make the gravity disturbance signal more noticeable from measurement errors and provide the detailed gravity field in sufficient resolution, decreasing the altitude of the vehicle is a direct and easy method ( $\mathrm{Li}, 2007)$. A possible option presented in this study is using Unmanned Aerial System (UAS), and the advantages include its high maneuverability, operation flexibility as well as capability to provide vector gravimetry results.

In this study, the proposed UAS has successfully integrated a navigation-grade IMU and GNSS for vector gravimetry. In the experiment, the performance evaluations of gravity disturbance vector to real flight data are discussed.

\footnotetext{
* Corresponding author
} 


\section{IMPLEMENTATION OF UAS}

In this study, the proposed UAS contains flight control and INS/GNSS integration. The communication distance can be 40 $\mathrm{km}$ and the raw measurements are logged for the navigation solution and gravimetry module.

\subsection{Specification of UAS platform}

The UAS is jointly developed by the Department of Geomatics, NCKU and AVIX Technology Inc. in Taiwan. As shown in Figure 1. Large payload space with easy installation and high mobility are main features. Table 1 shows the specifications of the UAS platform. The flexible flight altitude and one hour endurance time make it suitable for remote sensing and gravimetry in small area and high resolution.

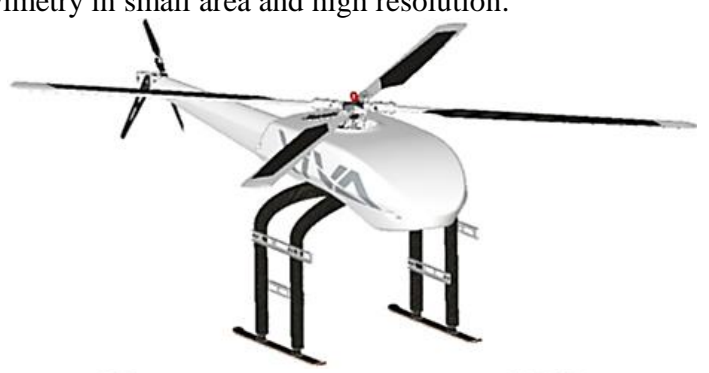

MAX. TAKE-OFF WEIGHT : 30KG

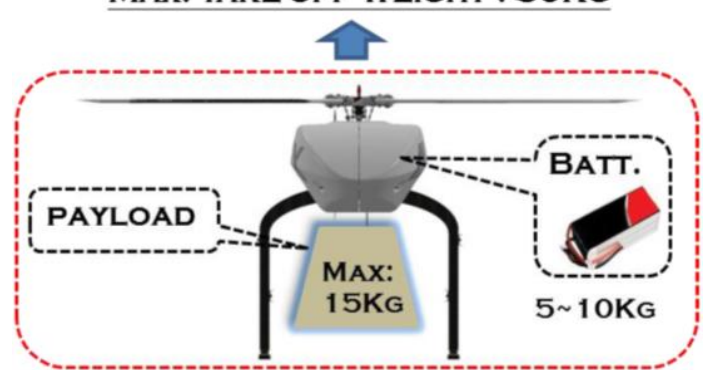

Figure 1. The UAS platform developed for gravimetric application

\begin{tabular}{|l|c|}
\hline & Value \\
\hline Only UAS Weight & $9 \mathrm{~kg}$ \\
Max. Take-off Weight & $30 \mathrm{~kg}$ \\
Battery Weight & $5 \sim 10 \mathrm{~kg}$ \\
Payload & $15 \mathrm{~kg}$ \\
Max. Operational Range & $40 \mathrm{~km}$ \\
Max. Cruise Speed & $85 \mathrm{~km} / \mathrm{h}$ \\
Max. Endurance & $1 \mathrm{~h}$ \\
\hline \multicolumn{2}{|c|}{ Table 1. The specifications of UAS platform }
\end{tabular}

\subsection{Configuration of flight control module}

For the autopilot module of proposed UAS platform, the advances neural network and adaptive theory are adopted in the basic control logic to perform the inertial autonomous navigation control. The use of GNSS aiding, Real Time Kinematic (RTK) technique, and ground control station, the control accuracy can achieve the centimeter-level in position.

The ground control station equips with a built-in $900 \mathrm{MHz}$ data link module to keep the real-time communication for autonomous navigation flight mission. Besides, through the data link module, the flight status information can be monitored on the screen and revise the post-flight mission.

\subsection{INS/GNSS integration with RTS smoother}

In this study, INS and GNSS measurements are used to provide navigation solutions and estimate gravity. The configuration is illustrated in Figure 2, the INS is tightly installed on the iron plate in the payload space and the GNSS antenna is mounted on the tailboom.

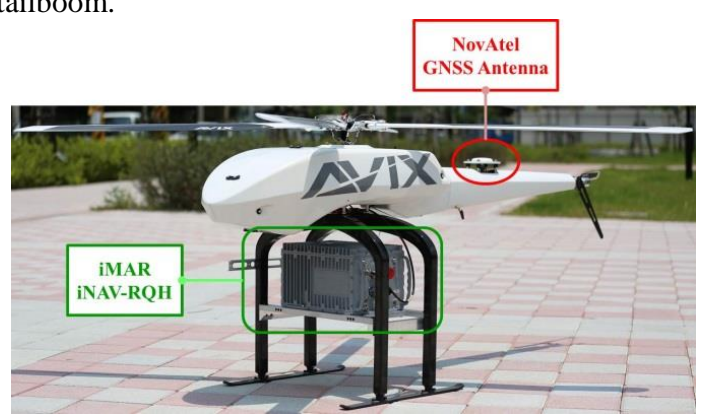

Figure 2. The configuration of INS and GNSS

The kinematic positions are post processed in this study. The GNSS antenna of the rover station is from NovAtel with $5 \mathrm{~Hz}$ sampling rate, which can receive L1/L2 GPS and G1/G2 GLONASS signals. The pulse per second signal is also sent to the INS for time synchronization.

The INS is a strapdown navigation-grade unit of type iNAV$\mathrm{RQH}$ with $300 \mathrm{~Hz}$ sampling rate from iMAR. It contains threeaxis accelerometers and three-axis ring laser gyroscopes, and the appearance is shown in Figure 3. Because the pure weight of the IMU is approximate 9 kilograms, proposed UAS platform is suitable for the purposes of this study. Table 2 lists the main performance characteristics of both sensors.

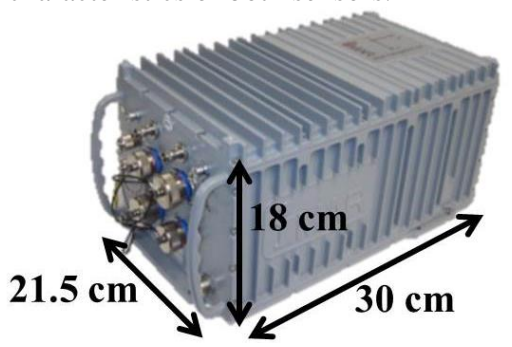

Figure 3. The exterior view and size of iNAV-RQH

The accurate navigation solution is extremely crucial for gravity estimation. Therefore, the tightly-coupled integration is implemented in this study to process INS and GNSS measurements by using Rauch-Tung-Striebel (RTS) smoother (Rauch et al., 1965). It can optimally estimate the system states, because the forward estimation is obtained using standard Kalman filter and the estimation of the backwards pass is based on the maximum likelihood estimates (Chiang et al., 2012).

\begin{tabular}{|l|c|c|}
\hline & Accelerometer & Gyroscope \\
\hline Measurement range & $\pm 20 \mathrm{~g}$ & $\pm 400^{\circ} / \mathrm{s}$ \\
Bias stability & $<10 \mu \mathrm{g}$ & $<0.002^{\circ} / \mathrm{hr}$ \\
Scale factor & $100 \mathrm{PPM}$ & $5 \mathrm{PPM}$ \\
Acceleration noise density & $8 \mu \mathrm{g} / \sqrt{\mathrm{Hz}}$ & - \\
Angular random walk & - & $0.0015^{\circ} / \sqrt{\mathrm{hr}}$ \\
Non-linearity & $15 \mu \mathrm{g} / \mathrm{g}^{2}$ & $10 \mathrm{PPM}$ \\
Misalignment & \multicolumn{2}{|c|}{$<15 \mu \mathrm{rad}$} \\
\hline
\end{tabular}

Table 2. The performance characteristics of iNAV-RQH 


\section{VECTOR GRAVIMETRY DETERMINATION}

In this study, the proposed UAS is used for the vector gravimetry. INS measures the dynamic acceleration directly applied on lifting and dragging, and the position of UAS can be obtained from GNSS. Because all calculations are performed in the i-frame, the fundamental equation is based on Newton's second law of motion expressed in a non-rotating and free falling coordinate frame (Jekeli, 2001), which reads as follows:

$$
\mathrm{g}^{\mathrm{i}}=\mathrm{a}^{\mathrm{i}}-\ddot{\mathrm{X}}^{\mathrm{i}}
$$

where $\mathrm{g}^{\mathrm{i}}=$ gravity vector

$\mathrm{a}^{\mathrm{i}}=\mathrm{IMU}-$ measured dynamic acceleration

$\ddot{X}^{\mathrm{i}}=$ GNSS-derived kinematic acceleration

The gravity vector is determined by subtracting the IMUmeasured dynamic acceleration from GNSS-derived kinematic acceleration. The flowchart for the vector gravimetry determination is shown in Figure 4, and there are four main steps involved. Because high-accuracy INS-measured dynamic accelerations and GNSS-derived kinematic accelerations are crucial in obtaining mGal-level gravity disturbance estimations, B-spline smoother is applied as the low-pass filter in this study.

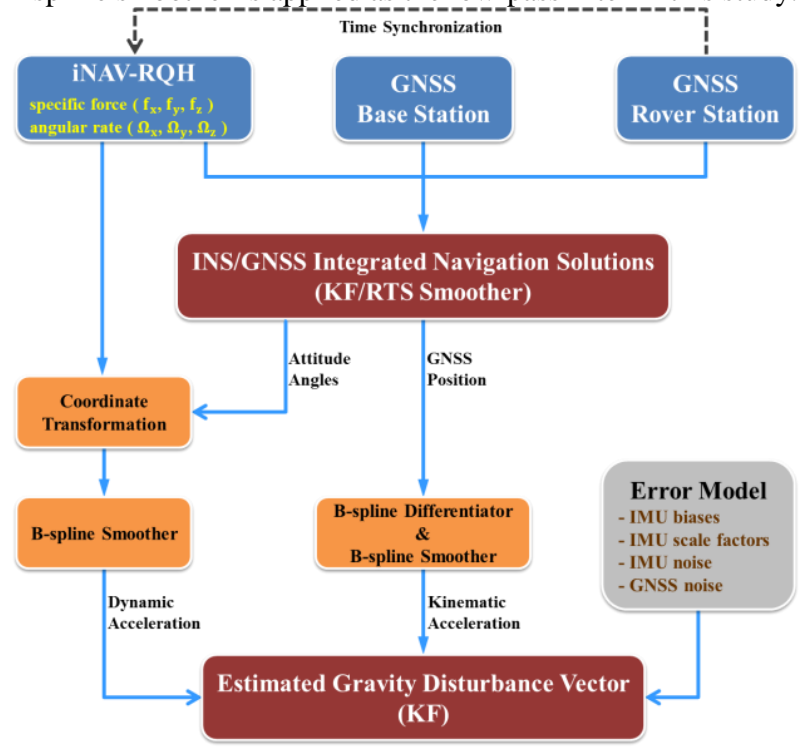

Figure 4. The determination flowchart of INS/GNSS gravimetry

First, the tightly-coupled integration of iNAV-RQH and GNSS measurements is processed by using RTS smoother. The optimal results of GNSS positions and attitude angles are obtained for further processing. Second, the kinematic accelerations are computed using the GNSS positions by applying a fifth-order B-spline differentiator. A third-order Bspline smoother with a window length of 60 seconds is adopted to remove high-frequency noises. The raw data of accelerometers from iNAV-RQH contain gravity and vehicle accelerations. The INS-measured dynamic accelerations are also processed by the same third-order B-spline smoother for consistency. Finally, the INS systematic errors are estimated and subsequently removed by the Kalman filter for estimating the gravity disturbance vector.

According to the previous study (Kwon and Jekeli, 2001), the GNSS-derived kinematic accelerations are used as updates. In addition, the residuals derived from the Kalman filter are assumed to approximate the gravity disturbance. The error states of the system are limited to the INS error parameters and the orientation errors, which link the accelerometer and gyroscope errors. The sensor errors comprise biases $\left(b_{a}, b_{g}\right)$ and scale factors $\left(f_{a}, f_{g}\right)$ with white noise $\left(\varepsilon_{a}, \varepsilon_{g}\right)$ (Kwon and Jekeli, 2001):

$$
\begin{aligned}
& \delta a^{b}=b_{a}+\left[a^{b}\right] f_{a}+\varepsilon_{a} \\
& \delta \omega_{i b}^{b}=b_{g}+\left[\omega_{i b}^{b}\right] f_{g}+\varepsilon_{g}
\end{aligned}
$$

where $\left[\mathrm{a}^{\mathrm{b}}\right],\left[\omega_{\mathrm{ib}}^{\mathrm{b}}\right]=$ diagonal matrices

$$
\omega_{\mathrm{ib}}^{\mathrm{b}}=\text { angular rate }
$$

The diagonal matrices are with diagonal elements equal to the elements of the indicated vectors, and the angular rate is of the INS body frame respect to i-frame. In total, the presented dynamics of the system states can be expressed as a first-order linear differential equation:

$$
\dot{\mathrm{s}}=\mathrm{Fs}+\mathrm{Gw}
$$

The matrix G properly scales the white noise vector (w), and the dynamic matrix $\mathrm{F}$ depends on the dynamics of the error state (s). The observation for the Kalman filter is a combination of the kinematic acceleration calculated from the GNSS position and normal gravity. The corresponding observed value to the dynamic acceleration is given by:

$$
\mathrm{y}=\tilde{\mathrm{a}}^{\mathrm{i}}-\left(\tilde{\ddot{\mathrm{X}}}^{\mathrm{i}}+\gamma^{\mathrm{i}}\right)
$$

On the basis of Equations (5) and (6), the state vector can be estimated by Kalman filter. The residual $(v)$ of the adjusted observation $(\hat{y})$ relative to the observed value is given by:

$$
\mathrm{v}=\mathrm{y}-\hat{\mathrm{y}}=-\delta \mathrm{g}^{\mathrm{i}}+\mathrm{R}_{\mathrm{b}}^{\mathrm{i}}\left(\delta \mathrm{b}_{\mathrm{a}}+\left[\mathrm{a}^{\mathrm{b}}\right] \delta \mathrm{f}_{\mathrm{a}}\right)-\tilde{\mathrm{a}}^{\mathrm{i}} \times \delta \dot{\Psi}^{\mathrm{i}}+\mathrm{R}_{\mathrm{b}}^{\mathrm{i}} \varepsilon_{\mathrm{a}}-\varepsilon_{\mathrm{g}}(7)
$$

The residual, computed by using Equation (7), contains the gravity disturbance vector as well as the true errors in the estimates of the system states and the noises. Compared to the gravity disturbance, the latter errors are relatively small. Therefore, the residual can be seen as an estimate of the gravity disturbance:

$$
v \approx-\delta g^{i}
$$

In this study, proposed algorithm of determining the gravity disturbance vector and estimating the IMU systematic errors is based on the technique of detecting outliers in a least-squares adjustment by inspecting the residuals corresponding to the adjusted observations.

\section{RESULTS AND DISCUSSIONS}

The performance of developed INS/GNSS gravimetry has been verified by using the land-vehicle system (Chiang et al., 2015). In this study, two kinematic tests were simultaneously conducted in an open sky area to evaluate vector gravimetry from the developed UAS. The flight altitude is approximately 25 meters with a speed of $25.2 \mathrm{~km}$ per hour. Figure 5 illustrates the comparisons between the smoothed INS and GNSS accelerations in $\mathrm{n}$-frame after normal gravity is removed. 

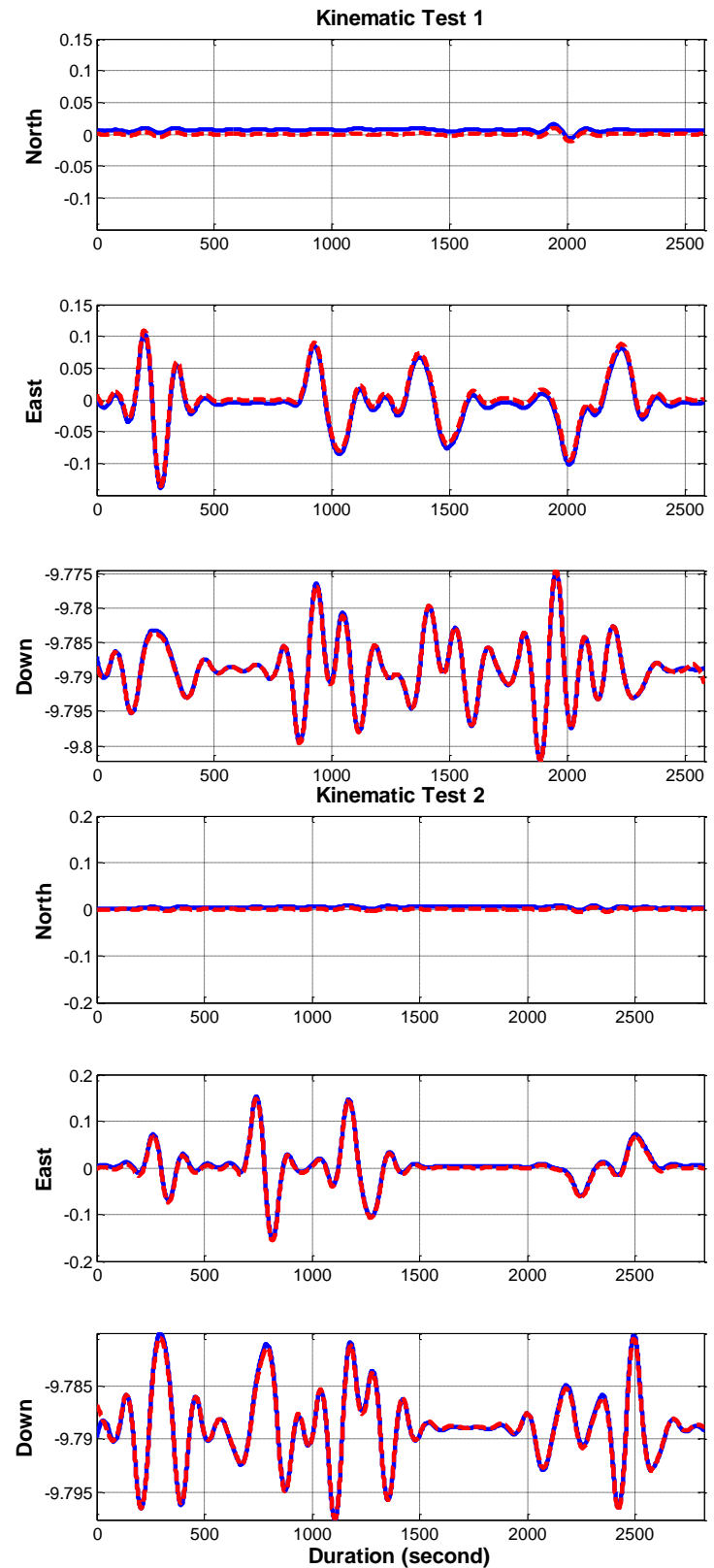

Figure 5. The accelerations $\left(\mathrm{m} / \mathrm{s}^{2}\right)$ derived from INS (blue) and GNSS (red) in n-frame

High-frequency oscillations of accelerations in the east-west component are stronger than those in the north-south component. This phenomenon is because the UAS platform is lighter than the aircraft; thus, the wind would significantly affect accelerations in the side direction. Moreover, the variations of the acceleration in the down component are reasonable. Therefore, both the smoothed results of INSmeasured dynamic accelerations and GNSS-derived kinematic accelerations correlate with the anticipated performances.

In the ideal state, the difference between the INS- and GNSSderived accelerations represents the gravity disturbance vector, according to Equation (2) and (6). However, the difference still contains systematic errors in three components. Figure 6 shows the large errors at hundred $\mathrm{mGal}$ level in the horizontal gravity disturbance derived by direct subtraction. By contrast, the results of estimating and removing the INS systematic errors by using Kalman filter have greatly improved.
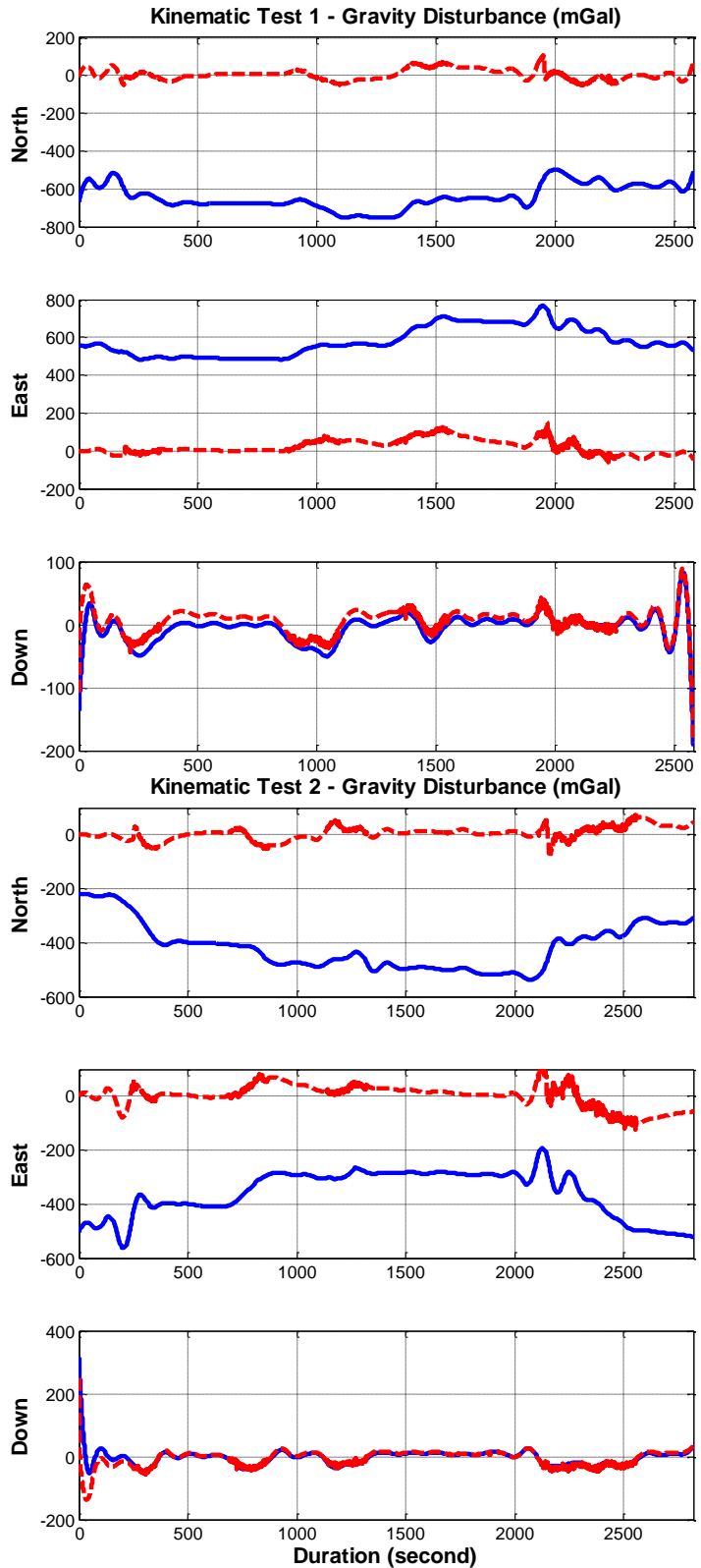

Figure 6 . Vector gravimetry determined by using Kalman filter (red) and derived from direct subtraction (blue)

As an indication of internal accuracy, the results of vector gravimetry estimated from the Kalman filter at crossover points are analyzed. The analysis used 38 crossover points (the distance of two points from two tests is less than $30 \mathrm{~cm}$ ). Table 3 shows the means and standard deviations (STDs) of the differences in the gravity disturbance vector at crossover points after the outliers have been removed to avoid the errors from the dynamic motion and environment. As expected, the internal accuracy in the down component is higher than that in the horizontal components because the orientation errors could cause large error in the horizontal components.

\begin{tabular}{|l|c|c|}
\hline \multirow{2}{*}{} & \multicolumn{2}{|c|}{ Difference in Gravity Disturbance (mGal) } \\
\cline { 2 - 3 } & Mean & STD \\
\hline North & 27.39 & 5.75 \\
East & -29.39 & 10.91 \\
Down & 22.70 & 4.04 \\
\hline
\end{tabular}

Table 3. The internal accuracy of INS/GNSS gravimetry with UAS at all crossover points 
Data processing indicates internal accuracies of approximately 4 $\mathrm{mGal}$ and $6-11 \mathrm{mGal}$ can be achieved for the vertical and horizontal components, respectively, with a spatial resolution of $0.5 \mathrm{~km}$. The estimated gravity disturbances in the down component are reasonable compared with the previously developed INS/GNSS airborne gravimetry which is in the range of $1-3 \mathrm{mGal}$ with a 2-km spatial resolution $(\mathrm{Li}, 2011)$.

Moreover, to evaluate the gravimetry results in the ZUPT mode, several artificial targets were planted as the ZUPT points along the road, as Figure 7 shows. In addition, Figure 8 illustrates the measure method presented in this study including the UAS performed automatic take-off and landing during the experiment, and was stopped at the ZUPT points to collect data.

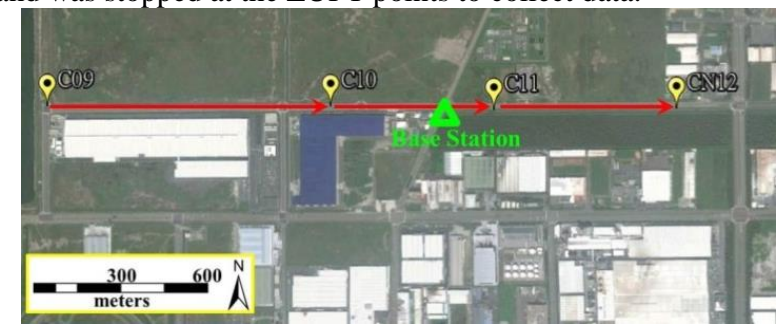

Figure 7. The experiment area and distribution of ZUPT points

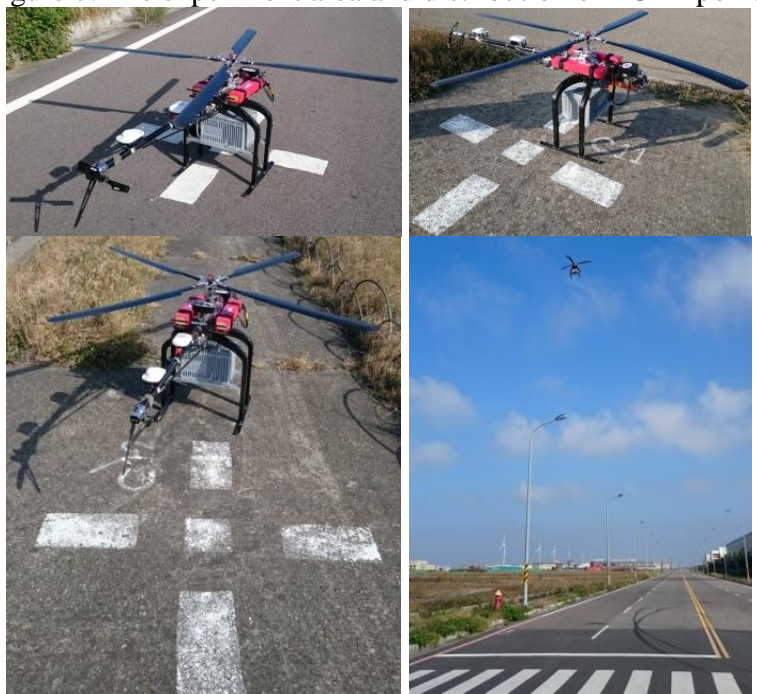

Figure 8 . The measure method in the experiment

The repeat measurements were conducted at the same ZUPT point and Table 4 lists the repeatability results of proposed UAS gravimetry in the ZUPT mode.

\begin{tabular}{|c|c|c|c|c|}
\hline & \multicolumn{2}{|c|}{$\begin{array}{l}\text { Gravity Disturbance } \\
\text { (mGal) }\end{array}$} & \multirow[t]{2}{*}{$\begin{array}{l}\text { Difference } \\
\text { (mGal) }\end{array}$} \\
\hline & & Test 1 & Test 2 & \\
\hline \multirow{3}{*}{ C09 } & $\mathrm{N}$ & 11.98 & 9.79 & 2.19 \\
\hline & $E$ & 0.18 & -0.15 & 0.33 \\
\hline & $\mathrm{D}$ & -2.42 & -2.28 & 0.14 \\
\hline \multirow{3}{*}{ C10 } & $\mathrm{N}$ & 6.65 & 4.56 & 2.09 \\
\hline & $E$ & -0.55 & -0.58 & 0.03 \\
\hline & $\mathrm{D}$ & 11.50 & 6.59 & 4.91 \\
\hline \multirow{3}{*}{ C11 } & $\mathrm{N}$ & -9.76 & -5.78 & 3.98 \\
\hline & $\mathrm{E}$ & 45.69 & 41.15 & 4.54 \\
\hline & D & 4.60 & 8.42 & 3.82 \\
\hline \multirow{3}{*}{$\mathrm{C} 12$} & $\mathrm{~N}$ & 8.41 & 8.47 & 0.06 \\
\hline & $\mathrm{E}$ & 25.05 & 24.35 & 0.70 \\
\hline & D & 10.14 & 13.91 & 3.77 \\
\hline
\end{tabular}

Table 4. The statistics of INS/GNSS gravimetry with UAS in the ZUPT mode
According to the preliminary results and analyses, the improved algorithms for determining and de-noising processing can make the system able for the precise gravimetry and geodetic applications. Moreover, according to the STDs of differences in three-dimension, the results of the gravity disturbance vector from INS/GNSS are reasonable with accuracies of about 2-3 mGal.

\section{CONCLUSIONS}

In this study, the INS/GNSS integration has been successfully carried by using a UAS for gravimetry. The determination algorithm is efficient and reliable for estimating the gravity disturbance vector.

The preliminary results of gravity disturbance from INS/GNSS data processing show that the accuracies at crossover points are approximately $4 \mathrm{mGal}$ and $6-11 \mathrm{mGal}$ in the vertical and horizontal components, respectively, with a $0.5-\mathrm{km}$ spatial resolution. In addition, the ZUPT mode implemented in this study is a novel measure method, the repeatability is evaluated with accuracies of about $2-3 \mathrm{mGal}$. Therefore, the capability of the developed UAS has been evaluated through various scenarios.

\section{ACKNOWLEDGEMENTS}

The authors would acknowledge the financial supports through the project funded by Ministry of Science and Technology (MOST 102-2221-E-006-137-MY3). In addition, we also thank the AVIX Technology Inc. for the assistance in developing the UAS and conducting the flight.

\section{REFERENCES}

Chiang, K.W., Duong, T. T., Liao, J.K., Lai, Y.C., Chang, C.C., Cai, J.M., and Huang, S.C., 2012. On-Line Smoothing for an Integrated Navigation System with Low-Cost MEMS Inertial Sensors. Sensors, 12(12), pp. 17372-17389.

Chiang, K.W., Lin, C.A., and Kuo, C.Y., 2015. A Feasibility Analysis of Land-Based SINS/GNSS Gravimetry for Groundwater Resource Detection in Taiwan. Sensors, 15(10), pp. 25039-25054.

Forsberg, R., and Olesen, A.V., 2010. Airborne Gravity Field Determination. In: Sciences of Geodesy - I: Advances and Future Directions, pp. 83-104.

Glennie, C.L., 1999. An analysis of airborne gravity by strapdown INS/DGPS. Ph.D. Thesis, Department of Geomatics Engineering, University of Calgary, Calgary, AB, Canada.

Jekeli, C., 2001. Inertial Navigation System with Geodetic Application; Walter de Gruyter: Berlin, Germany.

Kleusberg, A., Peyton, D., and Wells, D., 1990. Airborne Gravimetry and the Global Positioning System. In: Proceedings of Position Location and Navigation Symposium, pp. 273-278.

Kreye, C., and Hein, G.W., 2003. GNSS Based Kinematic Acceleration Determination for Airborne Vector Gravimetry Methods and Results, In: Proceedings of the 16th International Technical Meeting of the Satellite Division of The Institute of Navigation, pp. 2679-2691. 
Kwon, J.H., and Jekeli, C., 2001. A new approach for airborne vector gravimetry using GPS/INS. Journal of Geodesy, 74(10), pp. 690-700.

Li, X., 2007. Moving Base INS/GPS Vector Gravimetry on a Land Vehicle. Report No.486, Department of Geodetic Science and Surveying, The Ohio State University, Columbus Ohio, USA.

Li, X., 2011. Strapdown INS/DGPS airborne gravimetry tests in the Gulf of Mexico. Journal of Geodesy, 85(9), pp. 597-605.

Rauch, H., Tung, F., and Striebel, C., 1965. Maximum likelihood estimates of linear dynamic systems. AIAA Journal, 3, pp. $1445-1450$.

Schwarz, K.P., 2006. Simultaneous determination of position and gravity from INS/DGPS, Wissenschaftliche Arbeiten der Fachrichtung Geodaesie und Geoinformatik der Universitaet Hannover, Hannover, 258, pp. 141-148. 